\title{
A far-field based T-matrix method for three dimensional acoustic scattering
}

\author{
M. Ganesh ${ }^{1} \quad$ S. C. Hawkins ${ }^{2}$
}

(Received 14 August 2008; revised 4 October 2008)

\begin{abstract}
The acoustic scattering properties of an obstacle are completely described by its infinite acoustic T-matrix. The T-matrix is particularly useful when one is interested in analysing changes in sound wave propagation with respect to various changes in orientation or configuration of single or multiple scatterers. This is because the T-matrix is independent of the incoming wave directions and hence can be used to easily simulate the scattered sound waves, without the need to fully set up and solve each reconfigured system. However, in practice one must use the truncated finite dimensional T-matrix, which is usually computed using the null field method. For acoustically large obstacles or highly non-spherical particles the null field method is numerically unstable. In this work we describe an efficient and stable method for computing the truncated T-matrix using a surface integral equation reformulation and a high order acoustic surface scattering algorithm.
\end{abstract}

http://anziamj.austms.org.au/ojs/index.php/ANZIAMJ/article/view/1441 gives this article, (c) Austral. Mathematical Soc. 2008. Published October 27, 2008. ISSN 1446-8735. (Print two pages per sheet of paper.) 


\section{Contents}

1 Introduction

C122

2 Expansions of the acoustic field

C124

3 Far field based T-matrix computation

C127

4 Numerical experiments

C129

References

C135

\section{Introduction}

Acoustic scattering simulations using the T-matrix method, exterior to a ball circumscribing and centred inside the non-spherical scattering object, are based on series expansions of the incident and scattered fields using spherical wave functions. In sound wave propagation simulations the coefficients in the expansion of the input data (incident field) and the coefficients in the expansion of the output data (the scattered far-fields - processed through the Helmholtz operator) are connected by an infinite matrix, because the Helmholtz equation is linear. This transition matrix is called the T-matrix [2, $5,6,7]$. The acoustic scattering properties of an obstacle are completely described by its infinite acoustic T-matrix.

The T-matrix is a powerful tool when one is interested in scattering properties averaged over a range of incident directions because such information is readily obtained directly from the T-matrix. The T-matrix is also very useful for simulations of multiple scattering by ensembles of obstacles because the individual T-matrices of each scatterer can be combined using the translation-addition theorem $[5,6]$.

Waterman [9] intially developed the T-matrix for electromagnetic scatter- 
ing by a single scatterer; it was extended to multiple scatterers by Peterson and Ström [8]. The truncated T-matrix is usually computed using the null field (also known as the extended boundary condition) method [2, 4, 5, 9, 7].

For medium to high frequency problems or highly non-spherical obstacles the null field method is numerically unstable. This is largely due to fast growth of the spherical Hankel functions used in expansions of the surface field in the null field method [5]. For acoustically large or highly non-spherical obstacles the T-matrix computations can become divergent [7, p. 543]. There are several approaches to tackle this problem $[5,6,7]$ such as using expansions based on spheroidal or ellipsoidal functions for high-aspect ratio convex obstacles, and using slow extended precision arithmetic to minimise the effect of round-off errors.

The T-matrix could be computed using far field simulations based on surface integral equations for incident spherical waves, but to date no algorithms of this type have been developed for acoustic scattering in three dimensions [5, §7.9.4]. Many good algorithms exist for far field computations and such details are described by Ganesh and Graham [3]. This article focusses on the details required to compute the T-matrix by efficiently using these algorithms. In particular, we present details of an efficient fast stable scheme to compute the T-matrix entries. We assume that numerical far field patterns can be computed as necessary but we do not consider how to perform these far field computations here.

The fundamental computational difficulty in computing the T-matrix using a stable far field surface integral method is that this approach requires solving a large number of complex dense linear systems with a fixed scattering matrix (obtained by discretising the associated surface integral operator) but thousands of right hand sides (corresponding to each wave function used in expanding the incident field). Consequently, using a computational scattering algorithm that allows set up, storage, and LU-factorisation of the numerical scattering matrix is crucial. LU-factorisation is not practically possible for three dimensional scattering problems using low order schemes such 
as the standard boundary element methods, that usually require hundreds of thousands to millions of unknowns for low to medium frequency scattering for each incident direction. Such large systems require iterative solvers to avoid setting up and storing the scattering matrix and hence thousands of acoustic scattering problems are to be set up and solved for each term in the incident wave expansions separately. Ganesh and Graham [3] give computational complexity and CPU time requirements for three dimensional scattering problems.

Ganesh and Graham [3] describe a spectrally accurate three dimensional scattering algorithm that requires fewer than $10 \%$ of the unknowns than several established algorithms and hence is ideal for acoustic T-matrix computations, solving for thousands of terms in the incident field expansions using simple back and forward substitution techniques after storage of the LU-factorisation of the numerical scattering matrix, which is only computed once.

In this work we present a novel algorithm that uses numerically computed far field data to compute the acoustic T-matrix. We use the algorithm of Ganesh and Graham [3] to compute the far field data. In the next two sections we develop all of the details required for the T-matrix computations. Section 4 computationally demonstrates the high order accuracy of our acoustic T-matrix computations using several non-spherical obstacles with acoustic size $k a \approx 100$, where $k$ is the wavenumber of the incident wave and $a$ is the physical diameter of the scatterer.

\section{Expansions of the acoustic field}

The time-harmonic radiating acoustic velocity field $\boldsymbol{u}$ scattered by an impenetrable three dimensional scatterer $\mathrm{D}$ in a homogeneous medium satisfies the 
Helmholtz equation

$$
\Delta u(x)+k^{2} u(x)=0, \quad x \in \mathbb{R}^{3} \backslash \bar{D},
$$

where $k=2 \pi / \lambda$ is the wavenumber and $\lambda$ the wavelength, and the Sommerfeld radiation condition

$$
\lim _{|x| \rightarrow \infty}|x|\left(\frac{\partial u}{\partial x}-i k u\right)=0,
$$

where the limit holds uniformly in all directions $\hat{\boldsymbol{x}}=\boldsymbol{x} /|\boldsymbol{x}|$. The scattered field $u$ is induced by the incident field $u^{i}$ with wavenumber $k$ and in this work we assume that the obstacle is sound-soft, leading to the Dirichlet boundary condition

$$
u(x)=-u^{i}(x), \quad x \in \partial D .
$$

Extension of the algorithm to sound-hard and absorbing obstacles (that is, Neumann or mixed boundary conditions) is straightforward.

The T-matrix method is based on expansion of the incident and scattered fields in terms of spherical wavefunctions. In particular, the basic tool for expansions are the regular spherical wavefunctions

$$
\tilde{e}_{l j}(x)=j_{l}(k|x|) Y_{l j}(\hat{x}), \quad \hat{x}=\frac{x}{|x|},
$$

and radiating spherical wavefunctions

$$
e_{\mathrm{lj}}(\boldsymbol{x})=h_{\mathrm{l}}^{(1)}(k|x|) Y_{\mathrm{lj}}(\hat{\boldsymbol{x}}), \quad \hat{x}=\frac{\boldsymbol{x}}{|\boldsymbol{x}|},
$$

where $j_{l}$ is the spherical Bessel function of degree $l, h_{l}^{(1)}$ is the spherical Hankel function of degree $l$, and $Y_{l j}$ is the spherical harmonic of degree $l$ :

$$
Y_{l j}(\hat{x})=(-1)^{(j+|j|) / 2} \sqrt{\frac{2 l+1}{4 \pi} \frac{(l-|j|) !}{(l+|j|) !}} P_{l}^{|j|}(\cos \theta) e^{i j \phi},
$$


for $l=0, \ldots, \infty,|j| \leq l$, where $P_{l}^{|j|}$ is an associated Legendre function. Here we use the spherical polar coordinates representation of the unit vector $\hat{\chi}$ with polar angle $\theta$ and azimuth $\phi$. The spherical wavefunctions satisfy the Helmholtz equation (1). The radiating spherical wavefunctions additionally satisfy the radiation condition (2), which is an important constraint on the scattered acoustic fields that guarantees their outgoing nature.

We expand the given incident field as

$$
u^{i}(\boldsymbol{x})=\sum_{l=0}^{\infty} \sum_{|j| \leq l} p_{l j} \tilde{e}_{l j}(\boldsymbol{x}),
$$

with coefficients $p_{\mathfrak{l}}$, which are given analytically when $\mathfrak{u}^{\mathrm{i}}$ is a plane wave. The T-matrix technique assumes a similar expansion of the outgoing scattered exterior wave field:

$$
u(x)=\sum_{l=0}^{\infty} \sum_{|j| \leq l} a_{l j} e_{l j}(x) .
$$

Since the Helmholtz equation (1) (which connects the incident and scattered fields) is linear, there exist coefficients $t_{l^{\prime} j^{\prime}, l, j}$ such that

$$
a_{l^{\prime} j^{\prime}}=\sum_{l=0}^{\infty} \sum_{|j| \leq l} t_{l^{\prime} j^{\prime}, l j} p_{l j} .
$$

Writing $\boldsymbol{a}=\left(\boldsymbol{a}_{\mathrm{lj}}\right)$ and $\boldsymbol{p}=\left(\mathrm{p}_{\mathrm{lj}}\right)$, we have

$$
\mathbf{a}=\mathrm{Tp}
$$

where $T=\left(t_{l^{\prime} j^{\prime}, l j}\right)$ is called the transition- or T-matrix. 


\section{$3 \quad$ Far field based T-matrix computation}

A given radiating acoustic field $u$ has far field $u^{\infty}$ and acoustic cross section $\epsilon^{\mathrm{dB}}$ (measured in decibels), defined by

$$
u^{\infty}(\hat{x})=\lim _{|\boldsymbol{x}| \rightarrow \infty}|\boldsymbol{x}| e^{-i k|x|} \mathfrak{u}(\boldsymbol{x}), \quad \epsilon^{\mathrm{dB}}(\hat{\boldsymbol{x}})=10 \log _{10} 4 \pi\left|u^{\infty}(\hat{x})\right|^{2}, \quad \hat{x}=\frac{\boldsymbol{x}}{|\boldsymbol{x}|},
$$

which describes the asymptotic behaviour of the exterior field at large distances from the scatterer. This section describes an efficient computational scheme for computing the T-matrix of D using the far fields of the radiating spherical wavefunctions.

The scattered field $u$ is induced by the incident field through the boundary condition (3). Applying the operator that maps incident fields to scattered fields in the representation (7) for the incident field we get

$$
u=\sum_{l=0}^{\infty} \sum_{|j| \leq l} p_{l j} u_{l j}
$$

where $\mathfrak{u}_{\mathfrak{l j}}$ denotes the scattered field induced by $\tilde{e}_{\mathfrak{l j}}$. Each $\mathfrak{u}_{\mathrm{lj}}$ is a radiating solution of (1) and so has an expansion in the radiating spherical wavefunctions,

$$
u_{l j}=\sum_{l^{\prime}=0}^{\infty} \sum_{\left|j^{\prime}\right| \leq l^{\prime}} t_{l^{\prime} j^{\prime}, l j} e_{l^{\prime} j^{\prime}}, \quad l=0, \ldots, \infty,|j| \leq l,
$$

for some coefficients $t_{l^{\prime} j^{\prime}, l j}$.

From the asymptotics [1] of the spherical Hankel functions $h_{l}^{(1)}$, the far field $e_{l^{\prime} j^{\prime}}^{\infty}$ of the radiating spherical wavefunction $e_{l^{\prime} j^{\prime}}$ is

$$
e_{l^{\prime} j^{\prime}}^{\infty}(\hat{x})=\frac{1}{k}(-i)^{l^{\prime}+1} Y_{l^{\prime}, j^{\prime}}(\hat{x})
$$


Using (13) and (12) we derive the far field $\mathfrak{u}_{\mathfrak{l j}}^{\infty}$ of $\mathfrak{u}_{\mathfrak{l j}}$

$$
u_{l_{j}}^{\infty}=\sum_{l^{\prime}=0}^{\infty} \sum_{\left|j^{\prime}\right| \leq l^{\prime}} t_{l^{\prime} j^{\prime}, l j} \frac{1}{k}(-i)^{l^{\prime}+1} Y_{l^{\prime}, j^{\prime}}(\hat{x}) .
$$

Using the orthogonality of the spherical harmonics (6) we pick out the Tmatrix entries from a numerically computed approximation to $\mathfrak{u}_{\mathfrak{l j}}^{\infty}$ using the inner product

$$
t_{l^{\prime} j^{\prime}, l j}=\left\langle u_{l j}^{\infty}, Y_{l^{\prime} j^{\prime}}\right\rangle
$$

where $\langle\cdot, \cdot\rangle$ is the usual inner product on the unit sphere.

For practical computations the infinite T-matrix must be truncated, so that we restrict to wavefunctions of order $l, l^{\prime}=0, \ldots, n_{t}$ in all expansions. That is, we replace the symbol $\infty$ in all of the above infinite sums with a parameter $n_{\mathrm{t}}$. In practice we evaluate the inner product (15) using a GaussLegendre quadrature scheme [3] of order $2\left(n_{t}+1\right) \times\left(n_{t}+1\right)$,

$$
\int_{\partial B} f(\hat{x}) d s(\hat{\chi}) \approx \sum_{r=1}^{2 n_{t}+2} \sum_{s=0}^{n_{t}} \mu_{s} v_{r} f\left(\hat{\chi}_{r s}\right)
$$

where in spherical polar coordinates $\hat{\chi}_{\mathrm{rs}}=\hat{\boldsymbol{\chi}}\left(\theta_{\mathrm{r}}, \phi_{\mathrm{s}}\right)$ and $\nu_{\mathrm{s}}$ and $\mu_{\mathrm{r}}$ are quadrature weights. This quadrature scheme converges super-algebraically for smooth functions on the sphere such as those in (15).

The tensor product nature of the quadrature rule (16) allows us to take advantage of the separable nature of the spherical harmonics in the computations. For each $l, j$ we first precompute the array

$$
B_{s j^{\prime}, l j}=\sum_{r=1}^{2 n_{t}+2} \mu_{r} u_{l j}^{\infty}\left(\hat{x}_{r s}\right) e^{-i j^{\prime} \phi_{r}},
$$

and then compute

$$
t_{l^{\prime} j^{\prime}, l j}=\left.\sum_{s=0}^{n_{t}} v_{s} c_{j^{\prime}}^{l^{\prime}}\right|_{l^{\prime}} ^{\left|j^{\prime}\right|}\left(\cos \theta_{s}\right) B_{s j^{\prime}, l j}
$$


where $c_{j^{\prime}}^{l^{\prime}}$ is the normalisation coefficient in (6). This reduces the complexity of computing the T-matrix from $\mathrm{O}\left(n_{t}^{6}\right)$ to $\mathrm{O}\left(n_{t}^{5}\right)$. We further reduce the complexity by using the FFT to compute $\mathrm{B}_{\mathrm{sj}^{\prime}, \mathfrak{l j}}$.

To compute the T-matrix we must perform one far field computation to compute an approximation to the far field $\mathfrak{u}_{\mathfrak{l j}}^{\infty}$ for each incident wavefunction $\tilde{e}_{l j}, l=0, \ldots, n_{t},|j| \leq l$. For the numerical experiments in Section 4 we use the surface integral equation algorithm of Ganesh and Graham [3] because the high order basis used leads to small linear systems that we solve very efficiently using LU-factorisation, even for many right-hand sides induced by the many incident wavefunctions.

\section{$4 \quad$ Numerical experiments}

We demonstrate the convergence and accuracy of our algorithm by measuring symmetry properties of our computed truncated T-matrix for a range of convex and non-convex perfect conductors at low to medium frequencies. Figures 1-4 visually demonstrate the algorithm with exterior field and acoustic cross section computations, using our computed T-matrix.

The exact infinite T-matrix satisfies [5, Equation (7.67), p. 269]

$$
0=\sigma_{l^{\prime} j^{\prime}, l j}:=\mathrm{t}_{l^{\prime} j^{\prime}, l j}+\overline{\mathrm{t}_{l j, l^{\prime} j^{\prime}}}+2 \sum_{\hat{\imath}=0}^{\infty} \sum_{|\hat{\jmath}| \leq \hat{\imath}} \overline{\mathrm{t}_{\hat{\imath}, l^{\prime} j^{\prime}}} t_{\hat{\imath}, \mathfrak{l}, \mathfrak{j}} .
$$

This condition, with the summation appropriately truncated, is a standard measure of the quality of a numerically computed T-matrix [5, p. 269]. In this work we replace the infinite sum in (17) with $\sum_{\hat{\imath}=0}^{n_{t}} \sum_{|\hat{\jmath}| \leq \hat{\imath}}$ and use

$$
\sigma:=\max _{l=0, \ldots, n_{\mathrm{t}}} \max _{|j| \leq l} \max _{l^{\prime}=0, \ldots, n_{\mathrm{t}}} \max _{\left|j^{\prime}\right| \leq l^{\prime}}\left|\sigma_{l^{\prime} j^{\prime}, \mathrm{lj}}\right|
$$




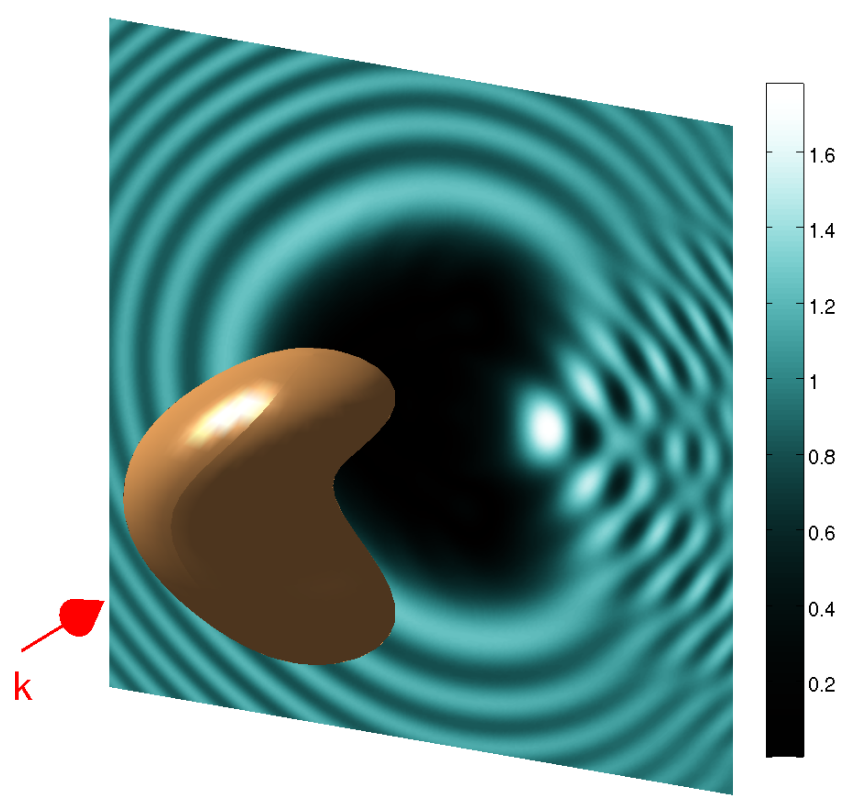

FiguRE 1: Intensity of the exterior field $(|\mathfrak{R}(\mathfrak{u})|)$ and detection of radiation free (shadow) region behind the bean shaped obstacle of acoustic size $\mathrm{ka}=$ 100.5310 computed using the T-matrix with $n=95$ and $n_{t}=75$. 


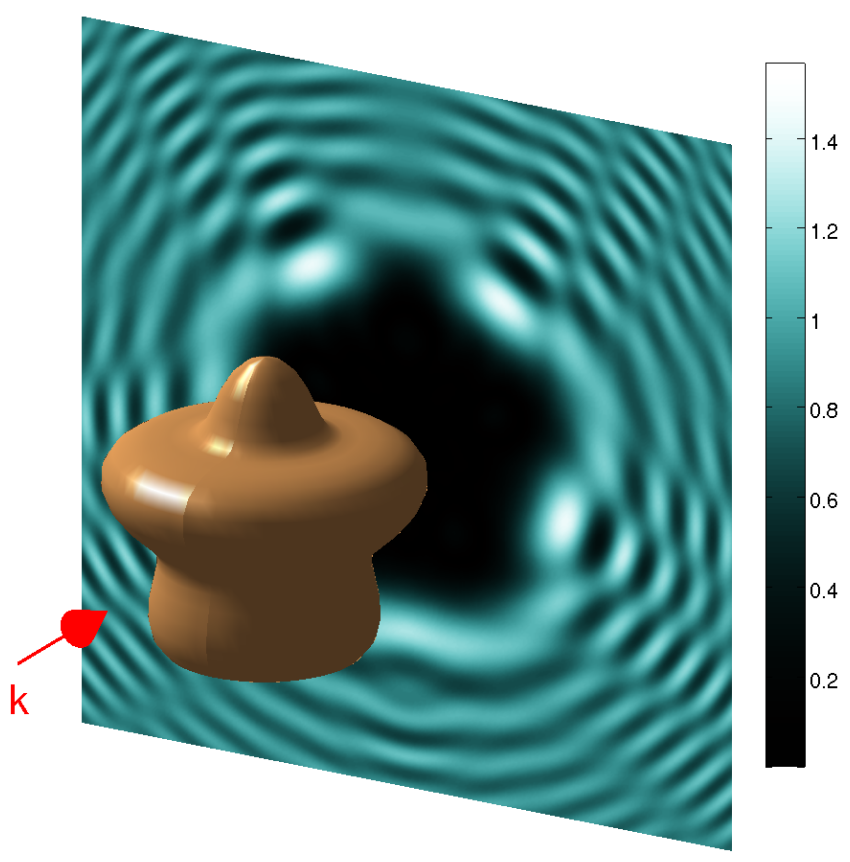

FiguRE 2: Intensity of the exterior field $(|\mathfrak{R}(\mathfrak{u})|)$ and detection of radiation free (shadow) region behind the fountain shaped obstacle of acoustic size $k a=100.5310$ computed using the T-matrix with $n=110$ and $n_{t}=75$. 

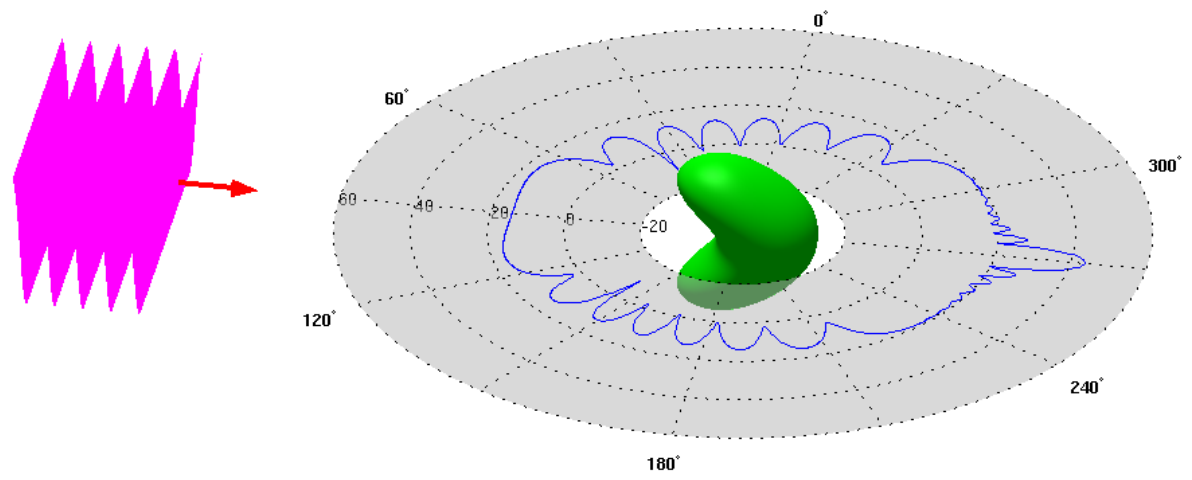

FiguRE 3: Bistatic acoustic cross section for a non-convex bean shaped obstacle of acoustic size $\mathrm{ka}=100.5310$ computed using the T-matrix with $n=95$ and $n_{t}=75$.
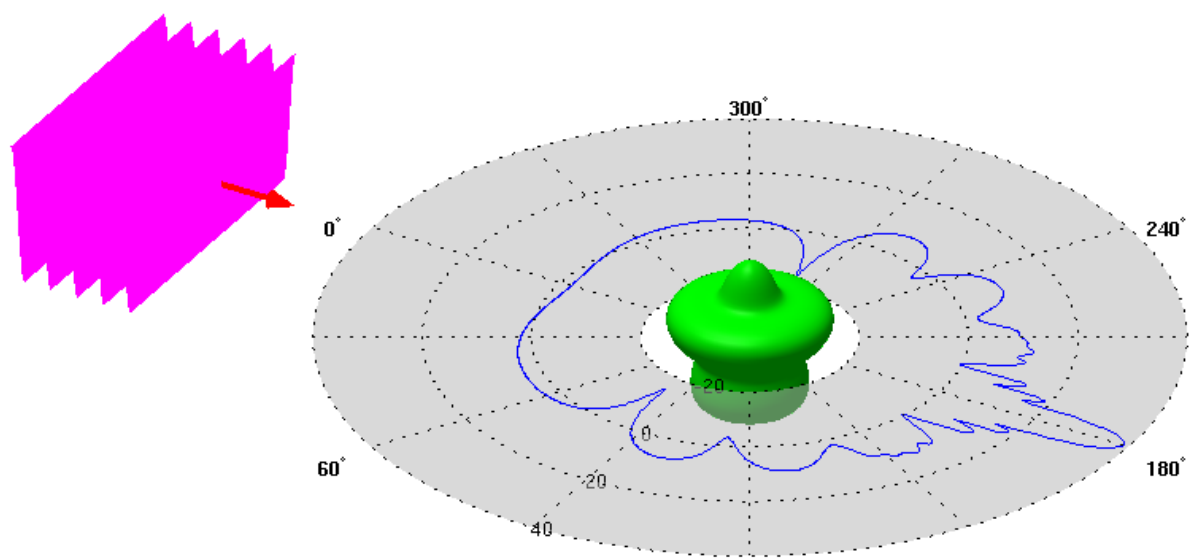

$120^{\circ}$

FiguRE 4: Bistatic acoustic cross section for a non-convex fountain shaped obstacle of acoustic size $\mathrm{ka}=100.5310$ computed using the T-matrix with $n=110$ and $n_{t}=75$. 

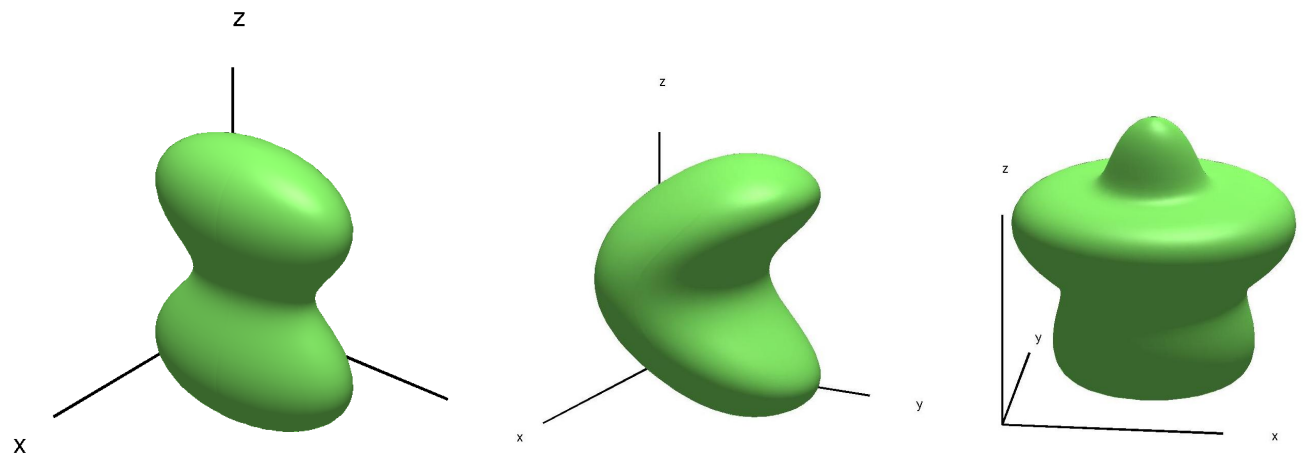

Figure 5: Non-convex peanut, bean and fountain shaped scatterers.

TABLE 1: Accuracy of T-matrix, measured by convergence of $\sigma$ in (18) to zero, for scattering by a peanut shaped obstacle with $\mathrm{ka}=100.5310$.

\begin{tabular}{cccc}
\hline $\mathrm{n}_{\mathrm{t}}$ & $\mathrm{n}=125$ & $\mathrm{n}=130$ & $\mathrm{n}=135$ \\
\hline 65 & $5.7 \mathrm{e}-07$ & $5.8 \mathrm{e}-07$ & $5.8 \mathrm{e}-07$ \\
70 & $2.8 \mathrm{e}-08$ & $5.0 \mathrm{e}-09$ & $4.8 \mathrm{e}-09$ \\
75 & $2.8 \mathrm{e}-08$ & $1.3 \mathrm{e}-09$ & $5.1 \mathrm{e}-11$ \\
\hline
\end{tabular}

as a measure of the error in our truncated T-matrix. A nice feature of $\sigma$ is that it measures errors due to truncation of the T-matrix as well as errors in the computation of the T-matrix entries.

In our numerical experiments we compute the far field using Ganesh and Graham's algorithm [3], which has spectral convergence with respect to the degree $\boldsymbol{n}$ of the approximating polynomials. In particular, for the algorithm, the size of the scattering matrix is $\mathrm{N} \times \mathrm{N}$, where $\mathrm{N}=(\mathrm{n}+1)^{2}$. For a given scatterer and incident wavenumber, the $\mathrm{n}$ required depends on the acoustic size $\mathrm{ka}$ and the shape of the scatterer. Ganesh and Graham [3] give full details for the choice of $n$. The shapes of some of the non-spherical obstacles considered in our experiments are shown in Figure 5. For these non-convex obstacles, as demonstrated in Tables $1-3$ with $\mathrm{ka} \approx 100$, it is sufficient for $\mathrm{n}$ to be at most 135, leading to scattering matrices of dimension less 
TABLE 2: Accuracy of T-matrix, measured by convergence of $\sigma$ in (18) to zero, for scattering by a bean shaped obstacle with $\mathrm{ka}=100.5310$.

\begin{tabular}{cccc}
\hline $\mathrm{n}_{\mathrm{t}}$ & $\mathrm{n}=85$ & $\mathrm{n}=90$ & $\mathrm{n}=95$ \\
\hline 65 & $7.3 \mathrm{e}-05$ & $7.3 \mathrm{e}-05$ & $7.3 \mathrm{e}-05$ \\
70 & $1.6 \mathrm{e}-06$ & $1.5 \mathrm{e}-06$ & $1.5 \mathrm{e}-06$ \\
75 & $1.6 \mathrm{e}-06$ & $1.2 \mathrm{e}-07$ & $2.0 \mathrm{e}-08$ \\
\hline
\end{tabular}

TABLE 3: Accuracy of T-matrix, measured by convergence of $\sigma$ in (18) to zero, for scattering by a fountain shaped obstacle with $\mathrm{ka}=100.5310$.

\begin{tabular}{cccc}
\hline $\mathrm{n}_{\mathrm{t}}$ & $\mathrm{n}=100$ & $\mathrm{n}=105$ & $\mathrm{n}=110$ \\
\hline 65 & $2.8 \mathrm{e}-06$ & $2.8 \mathrm{e}-06$ & $2.8 \mathrm{e}-06$ \\
70 & $2.3 \mathrm{e}-08$ & $2.3 \mathrm{e}-08$ & $2.3 \mathrm{e}-08$ \\
75 & $3.3 \mathrm{e}-09$ & $2.4 \mathrm{e}-10$ & $1.3 \mathrm{e}-10$ \\
\hline
\end{tabular}

than 18,500 . Such small systems allow us to solve using the LU-factorisation (in serial or parallel) for many right hand sides at very little extra cost.

We demonstrate the convergence of $\sigma$ in (18) to zero with respect to the parameters $n_{t}$ and $n$. For spherical scatterers with radius $r=a / 2$, the T-matrix is diagonal and the entries correspond to the well known series solution of the acoustic scattering problem. The $n_{t}$ required to obtain a good solution in this case is approximately $n_{t}=k r+4(k r)^{1 / 3}[10]$.

Our experimental results in Tables 1-3 suggest that for non-spherical scatterers slightly higher $n_{t}$ is required than for spherical scatterers to obtain $\sigma$ between $10^{-8}$ to $10^{-10}$. The results reported here are typical of the results obtained with our algorithm for a wide range of problems at low to medium frequency, with several non-spherical obstacles.

Acknowledgements Support of the Australian Research Council and the Colorado Golden Energy Computing Organization (GECO) is gratefully ac- 
knowledged. Computations were carried out using the GECO cluster RA.

\section{References}

[1] D. Colton and R. Kress. Inverse Acoustic and Electromagnetic Scattering Theory. Springer, 1998. C127

[2] A. Doicu, T. Wriedt, and Y. Eremin. Light Scattering by Systems of Particles. Null-Field Method with Discrete Sources-Theory and Programs. Springer Verlag, 2006. C122, C123

[3] M. Ganesh and I. G. Graham. A high-order algorithm for obstacle scattering in three dimensions. J. Comput. Phys., 198:211-242, 2004. doi:10.1016/j.jcp.2004.01.007. C123, C124, C128, C129, C133

[4] F. M. Kahnert. Numerical methods in electromagnetic scattering theory. J. Quant. Spectrosc. Radiat. Transfer, 79-80:775-824, 2003. doi:10.1016/S0022-4073(02)00321-7. C123

[5] P. A. Martin. Multiple Scattering: Interaction of Time-Harmonic Waves with $N$ Obstacles. Cambridge University Press, 2006. C122, C123, C129

[6] M. I. Mishchenko, L. D. Travis, and A. A. Lacis. Multiple Scattering of Light by Particles: Radiative Transfer and Coherent Backscattering. Cambridge University Press, 2006. C122, C123

[7] M. I. Mishchenko, L. D. Travis, and D. W. Mackowski. T-matrix computations of light scattering by nonspherical particles: a review. J. Quant. Spectrosc. Radiat. Transfer, 55:535-575, 1996. doi:10.1016/0022-4073(96)00002-7. C122, C123 
[8] B. Peterson and S. Ström. T matrix for electromagnetic scattering from an arbitrary number of scatterers and representations of $\mathrm{E}(3)$. Physical Review D, 8:3661-3678, 1973. doi:10.1103/PhysRevD.8.3661. C123

[9] P.C. Waterman. Matrix formulation of electromagnetic scattering. Proc. IEEE, 53:805-812, 1965. C122, C123

[10] W. J. Wiscombe. Improved Mie scattering algorithms. Applied Optics, 19, 1980. http:

//www.opticsinfobase.org/abstract.cfm?URI=ao-19-9-1505. $\mathrm{C} 134$

\section{Author addresses}

1. M. Ganesh, Department of Mathematical and Computer Sciences, Colorado School of Mines, Golden, CO 80401, USA mailto:mganesh@mines. edu

2. S. C. Hawkins, School of Mathematics and Statistics, University of New South Wales, Sydney, NSW 2052, Australia. mailto:stuart.hawkins@unsw.edu.au 